IN a recent paper in Astrophysical Journal $(235,625 ; 1980)$ H. Yoshimura has presented new results from computational simulations of non-linear astrophysical dynamos; in particular he has reproduced magnetic Field reversals. This represents a contribution to the theory of field generation (i.e. dynamo action) in a sphere of electrically conducting fluid, the object of such investigations being to explain the observed features of the magnetic fields of the Earth and the Sun. In the terrestrial context, it is necessary to explain why the magnetic field of the Earth survives in a quasi-steady state over geological time scales much longer than the natural decay time of the field (of order $10^{4}$ years). In the solar context, it is necessary to explain why the general magnetic field of the Sun evolves in an approximately periodic manner (with the same period as that of the sunspot cycle i.e. about 22 years) when the natural (ohmic) time scale for the Sun is of the order of $10^{9}$ years.

Traditional dynamo theory starts from coupled equations for the velocity field $\mathbf{u}$ and the magnetic field $\mathbf{B}$ in the fluid region

$$
\left.\begin{array}{l}
\frac{\delta \mathbf{B}}{\delta \mathrm{t}}-\lambda \nabla^{2} \mathbf{B}=\mathrm{L}\{\mathbf{u}, \mathbf{B}\} \\
\frac{\delta \mathbf{u}}{\delta \mathrm{t}}-\nu \nabla^{2} \mathbf{u}=\mathrm{N}\{\mathbf{u}, \mathbf{B}\}+\mathbf{F}
\end{array}\right\}
$$

In these equations $\mathrm{L}$ represents a differential operator that is linear in each of $\mathbf{u}$ and $\mathbf{B}, \mathbf{N}$ represents a nonlinear integral-differential operator, and $\mathbf{F}$ represents the force distribution, whatever this may be, which maintains the motion. Much controversy surrounds the nature of this force field in the terrestrial context, but in the solar H.K. Moffat is in the School of Mathematics, University of Bristol.

cavity and $\delta \mathrm{Z}$ is the longitudinal deviation from resonance, and $\Omega^{2} \alpha B_{o} \varepsilon$, the product of the wiggler field strength and the electric field amplitude of the generated radiation. The physics of electron bunches moving round storage rings is in general very complicated. However, as a first approximation, the ring system can also be modelled by a pendulum equation of the above type, where $\theta$ is now proportional to $\tau$, the time displacement of a test particle from the nominal position, and $\Omega^{2} \alpha \mathrm{V}$, the amplitude of the applied r.f. field.

For either system (laser or ring) taken in isolation, the above equation predicts trapped particle solutions, where the particles oscillate backwards and forwards in the potential wells determined by $\Omega-$ a phenomenon well known in plasma physics. The length scale of the potential wells (or 'buckets') will of course be different in the two systems, being very much shorter in the laser cavity where they

\title{
Non-linear astrophysical dynamos
}

\section{from H.K. Moffat}

context it is certainly of thermal origin.

For a given force field, one would like to solve these equations, and demonstrate the existence of solutions for $\mathbf{B}$ which have the observed property in either context. Unfortunately, this task is far beyond either analytical solution, or the power of present computers. Approximate techniques must be adopted in order to reduce the problem to a level that will yield to the computer. A first dramatic stage has been achieved over the last 20 years, with the realisation that when the velocity field $\mathbf{u}$ is turbulent, the mean magnetic field $\langle\mathbf{B}\rangle$ satisfies a simpler equation

$\left.\left(\frac{\delta}{\delta \mathrm{t}}-\lambda \nabla^{2}\right)<\mathbf{B}\right\rangle=\langle\mathrm{L}\rangle\langle\mathbf{B}\rangle(2)$

where $\langle\mathrm{L}\rangle$ is an averaged differential operator which is in principle determinate in terms of the statistical properties of the turbulence. For different forms of this averaged operator, solutions of equation (2) are known of types that capture the main features either of the Earth's quasisteady field, or of the Sun's quasiperiodic field.

The magnetic field itself influences the structure of the turbulence, through the second of Equations (1), and it is this "back reaction" effect that more recent theories are attempting to incorporate. First attempts in this direction were those of Stix (Astron. Astrophys. 20, 9; 1972) and Jepps (J. Fluid Mech. 67, 417; 1975), both of whom incorporated a fairly simple cut-off in the "regenerative" part of $\langle\mathrm{L}\rangle$ when the magnetic field exceeded

are of the order of the optical wavelength. If the two systems are now coupled, as shown in the figure, we have a very complicated system indeed comprising as it does two coupled highly nonlinear differential equations. Clearly, such a system can only be properly studied using numerical techniques.

In their preliminary study of the problem, Deacon and Madey proceed by linearising the above system and, in so doing, are able to establish that isochronous orbits will occur provided the operating parameters of the system fall within defined limits. Within these limits, the large operating efficiencies (>20\%) referred to earlier should be realised. It will be interesting to see whether a more complete (numerical) study of the problem confirms these preliminary results.

J.N. Elgin is in the Department of Physics, Imperial College, London. a level at which the back reaction effect was assumed to become significant. Yoshimura has developed this approach in a series of papers, in which he has considered increasingly sophisticated forms of the operator $\langle\mathrm{L}\rangle$, incorporating in particular a time-lag between the generation of a magnetic field and the (later) manifestation of its dynamic effects. This increasing sophistication in his models has enabled him to compute solutions of Equation (2) which resemble the observed fields of Earth and Sun in remarkable detail. As might perhaps be anticipated the more disposable parameters that are introduced in the assumed form of $\langle\mathrm{L}\rangle$, the more freedom there is at the end of the calculation to choose these parameters in a way that achieves agreement with observation. In particular, Yoshimura has now found solutions exhibiting reversals, like the welldocumented occasional reversal of the Earth's field.

While Yoshimura's approach does not increase physical insight at the fundamental level, it does lead to the accumulation of an interesting catalogue of possible behaviours of solutions of equations of the form (2), when time delay is incorporated in the operator $\langle\mathrm{L}\rangle$. The approach however is open to criticism in that all the subtleties of the dynamical equation (1b) are parametrized in what many would regard as an unacceptably crude manner. Alternative approaches (e.g. that advocated by Malkus \& Proctor (J. Fluid Mech. 67, $417 ; 1975$ ) treat the dynamics in a less brutal manner, and although such approaches have not yet been pushed as far (in terms of results) as Yoshimura's, they hold more promise in the longer term for providing a convincing theory of dynamo action including proper recognition of the role of dynamic effects.

It is perhaps worth while mentioning two alternative schemes to the laser-ring system discussed above, both of which have received some theoretical study. In the first of these, an attempt is again made to reverse the energy spread of the electrons, but this time using echo techniques. Here, the effect is similar to one obtained in nonlinear optics where a randomising process can be reversed by a suitable coherent interaction of an optical pulse with the system. The second alternative uses a tapered wiggler magnet to slow down the electron bunches trapped in the optical potential wells referred to earlier, with the loss in energy of the bunches going into the radiation field. This latter method would of course be single pass only. Each possible method has its advantages and disadvantages and, at the time of writing, it is not yet clear which (if any) will provide the means of obtaining high efficiencies in free-electron laser systems. 